\title{
Lipoma Cervicofacial Gigante: Reporte de un caso.
}

\section{Giant Cervicofacial Lipoma: A Case Report.}

\author{
Edgard Riquelme ${ }^{1}$, Tomás Aitken ${ }^{2}$, Benjamín Martínez ${ }^{3}$, Claudio San Martín ${ }^{4 *}$
}

1. Cirujano Dentista. Universidad Mayor, Santiago,

Chile.

2. Cirujano Dentista. Universidad de los Andes,

Santiago, Chile.

3. Académico, Departamento de Patología

y Medicina Oral, Facultad de Odontología,

Universidad Mayor, Santiago, Chile.

4. Académico, Departamento de Cirugía

y Traumatología Maxilofacial, Facultad de

Odontología, Universidad Mayor, Santiago, Chile.

* Correspondencia Autor: Claudio San Martín

Mardones | Dpto. Cirugía y Traumatología

Maxilofacial, Facultad de Odontología, Universidad

Mayor | Dirección: Avda. Libertador Bernardo

O'Higgins \#2013, Santiago, Chile. | Teléfono: +569

94190298 | E-mail: clausamar@vtr.net

Trabajo recibido el 17/05/2020.

Trabajo revisado 07/07/2020

Aprobado para su publicación el 18/07/2020

\section{RESUMEN}

Los lipomas son neoplasias benignas formadas por tejido adiposo maduro y que representan los tumores mesenquimales más frecuentes. Los lipomas gigantes se definen en relación a un tamaño mayor de 10 centímetros o un peso mayor a 1 kilogramo. Su presentación a nivel de cabeza y el cuello es infrecuente. Se caracterizan por tener un crecimiento lento, progresivo, deformante y asintomático. Es necesaria la realización de pruebas de imagen para su correcta localización anatómica y establecer relaciones con las estructuras vecinas. El tratamiento de elección es la escisión quirúrgica de la masa. La planificación pre-quirúrgica en este tipo de casos debe ser minuciosa para garantizar la conservación tanto de la función como de la estética cervicofacial. Se reporta el caso de una mujer de 50 años que presenta una tumoración gigante cervicofacial de 9 años de evolución, diagnosticada como lipoma, con sus características clínicas, imageneológicas, histopatológicas y su manejo terapéutico.

PALABRAS CLAVE:

Lipoma gigante; Masa cervical; Tumor mesenquimal.

Int. J. Inter. Dent Vol. 14(2); 181-183, 2021.

\section{ABSTRACT}

Lipomas are benign neoplasms formed by mature adipose tissue and represent the most frequent mesenchymal tumors. Giant lipomas are defined in relation to a size greater than 10 centimeters or a weight greater than 1 kilogram. Their presentation at head and neck level is infrequent. They are characterized by having a slow, progressive, deforming and asymptomatic growth. Imaging tests are necessary for their correct anatomical location and the establishment of relationships with neighboring structures. The treatment of choice is surgical excision of the mass. Pre-surgical planning in this type of case must be meticulous to guarantee the preservation of both function and cervicofacial aesthetics. The case of a 50-year-old woman with a giant cervicofacial tumor of 9 years of evolution, diagnosed as lipoma, with its clinical, imaging, histopathological characteristics, and therapeutic management is reported.

\section{KEY WORDS:}

Giant lipoma; Cervical mass; Mesenchymal tumor.

Int. J. Inter. Dent Vol. 14(2); 181-183, 2021.

\section{INTRODUCCIÓN}

Los lipomas son tumores benignos de tejido adiposo que representan las neoplasias de partes blandas más frecuentes. Tienen un origen mesenquimático e histológicamente se componen de células grasas maduras con múltiples subtipos patológicos ${ }^{(1)}$. Pueden aparecer en cualquier parte del cuerpo pero se desarrollan con más frecuencia en las extremidades y el tronco. Sin embargo, el $13 \%$ de los lipomas crecen en la región de la cabeza y el cuello(2).

Los lipomas de cabeza y cuello son más prevalentes en pacientes masculinos que femeninos en razón de 2,3:1. Se presentan generalmente entre la cuarta y sexta década de vida. Su localización de predilección es en la región frontal y postero-lateral del cuello ubicándose preferentemente en la capa grasa de la piel. Son lesiones bien circunscritas y tienen un tamaño promedio (en el eje mayor del tumor) de 31,92 (SD 18,87) milímetros ${ }^{(3)}$

Los lipomas comúnmente son lesiones solitarias y pequeñas que rara vez crecen hasta un tamaño excepcionalmente grande. Un lipoma se considera gigante cuando mide más de $10 \mathrm{~cm}$ en cualquier dimensión o pesa más de $1000 \mathrm{gm}^{(4)}$. Los lipomas gigantes (LG) se caracterizan por tener un crecimiento lento, progresivo, deformante y asintomático. Suelen encontrarse rodeados por una delgada cápsula fibrosa. Pueden desplazar estructuras anatómicas vecinas y confundirse con otras lesiones tumorales malignas $^{(5)}$

Presentamos el caso de una mujer de 50 años que presenta un LG en región cervical y facial derecha, describiendo sus características clínicas, imageneológicas, histopatológicas y su manejo terapéutico.

\section{INFORME DE CASO}

Mujer de 50 años de edad, con antecedentes de Diabetes Mellitus Tipo 2, consulta por aumento de volumen cervicofacial derecho, asintomático y de 9 años de evolución. Al examen clínico extraoral presenta aumento de volumen de consistencia blanda, no adherido a planos profundos (móvil), que abarca región geniana y cervical alta del lado derecho que provoca una considerable asimetría hemifacial (Figura 1). Al examen clínico intraoral se observa mucosa yugal en relación a la lesión transparentada de una coloración amarillenta. Paciente relata un crecimiento lento y progresivo de la lesión. No se palparon adenopatías ni otras masas.

Inicialmente se solicitó una Tomografía Computada (TC) con medio de contraste y exámenes de laboratorio (perfil hematológico, VHS, electrolitos séricos, glucosa y perfil de la coagulación) cuyos hallazgos fueron normales. En la TC se observó un aumento de volumen hipodenso, bien delimitado, encapsulado, que abarcaba región geniana, maseterina y cervical alta. Además, en la exploración de la TC de observó una extensión de la lesión a piso de boca (Figura 2). 

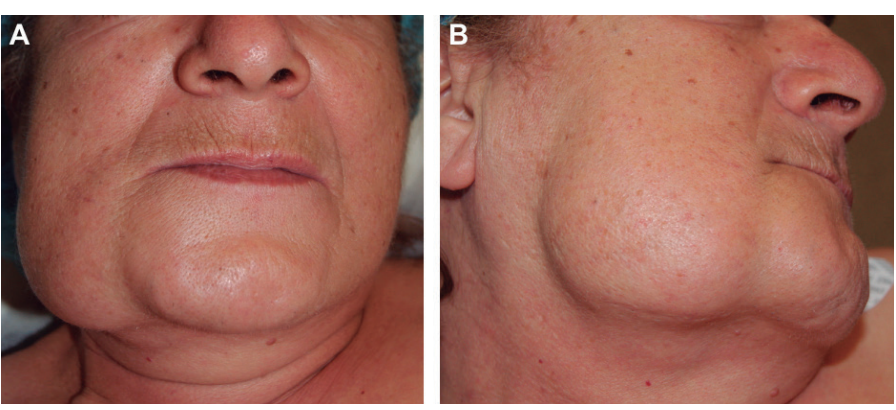

Figura 1. Vista frontal (A) y sagital (B) de examen facial. Aumento de volumen cervicofacial derecho
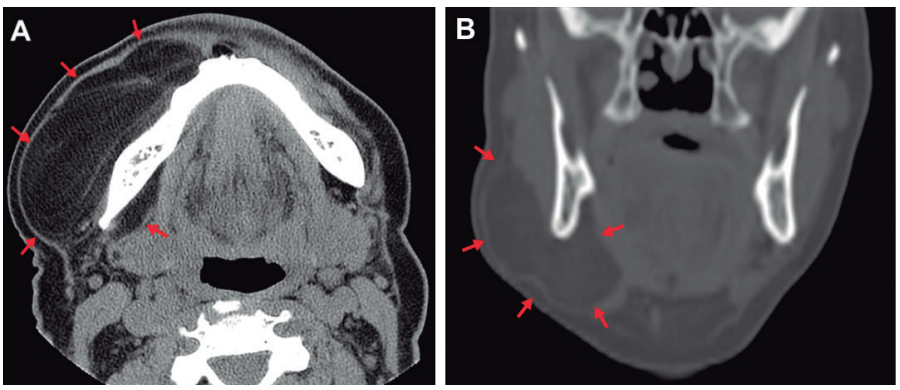

Figura 2. Tomografía Computada (TC) con medio de contraste. Cortes axial (A) y coronal (B) de cabeza y cuello donde se observan límites y extensión de la lesión tumoral (flechas)

Con los antecedentes clínicos y radiográficos obtenidos, se consideraron tres hipótesis diagnósticas: Mucocele, Lipoma y Quiste Dermoide.

Luego se realizó una punción aspirativa con aguja fina (PAAF) que confirmó las sospechas iniciales de lipoma.

El plan de tratamiento se llevó a cabo bajo anestesia general. La técnica quirúrgica se realizó vía abordaje cervical y vestibulotomía inferior derecha realizándose la exéresis y biopsia excisional de la lesión (Figura $3)$.

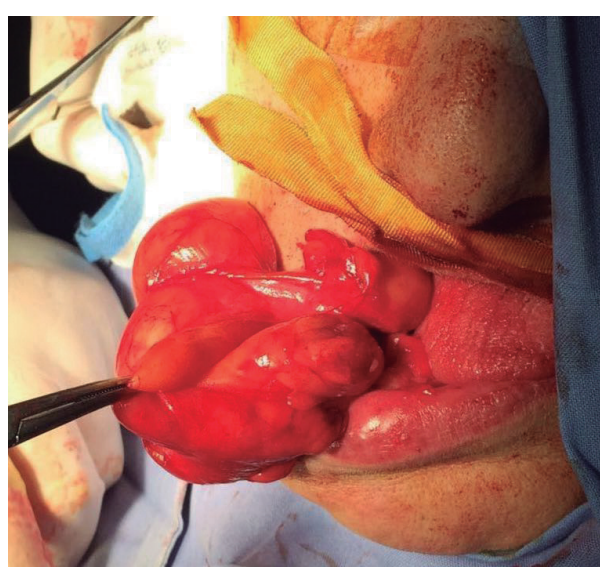

Figura 3. Imagen intraoperatoria de la exéresis de la lesión tumoral

Durante la intervención se obtuvo una pieza quirúrgica de 426,5 gramos de peso y unas dimensiones de $13 \times 6 \times 7,5$ centímetros en los ejes sagital, transversal y cráneo-caudal respectivamente.

Tras el segundo día postoperatorio la paciente fue dada de alta hospitalaria.

En el resultado de la histopatología se observó tejido adiposo adyacente a manojos de fibras colágenas, con algo de hialinización, pequeños capilares. Presencia de abundantes adipocitos de aspecto normal, separados por tabiques conjuntivales y pequeños capilares (Figura 4). En el informe histopatológico se confirmó el diagnóstico de Lipoma sin atipias.

Finalmente la paciente tras 6 meses de controles postoperatorios evolucionó satisfactoriamente y fue dada de alta de la especialidad.
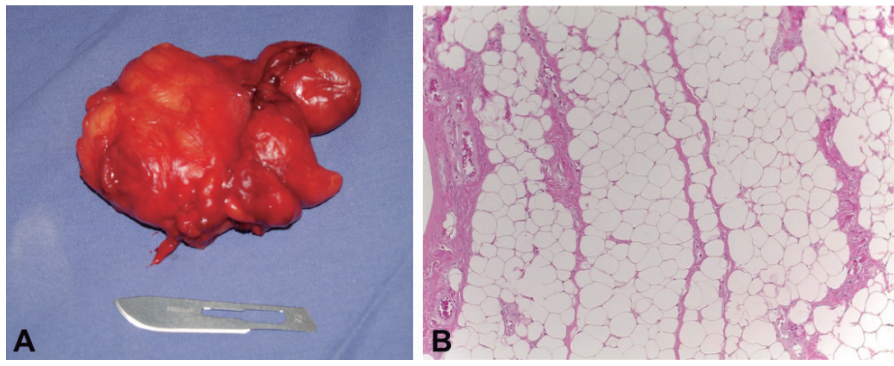

Figura 4. A. Muestra de tejido biopsiado B. Microfotografía de corte histológico tinción hemotoxilina-eosina x200. Se observa tejido adiposo adyacente a manojos de fibras colágenas, con algo de hialinización, pequeños capilares. Presencia de abundantes adipocitos de aspecto normal, separados por tabiques conjuntivales y pequeños capilares

\section{DISCUSIÓN}

Un lipoma se considera gigante cuando mide más de $10 \mathrm{~cm}$ en cualquier dimensión o pesa más de $1000 \mathrm{gm}^{(4)}$. Los LG se caracterizan por tener un crecimiento lento, progresivo, deformante y asintomático. Suelen encontrarse rodeados por una delgada cápsula fibrosa ${ }^{(5)}$. En nuestro caso, la tumoración abarcaba la región geniana, maseterina, cervical alta e incluso presentaba una prolongación a piso de boca. Su longitud en el eje mayor alcanzaba los $13 \mathrm{~cm}$; considerándose un LG como se describe en la literatura.

El mecanismo de crecimiento de los lipomas gigantes no está claro. Algunos estudios han postulado el papel del trauma como importante, sugiriendo que el trauma cerrado puede causar la ruptura de los tabiques fibrosos y las conexiones de anclaje entre la piel y la fascia profunda, permitiendo que el tejido adiposo prolifere rápidamente. También se ha sugerido que la liberación de citocinas inducida por el trauma, desencadena una diferenciación y maduración pre-adipocítica ${ }^{(6)}$.

Salvatore et al. ${ }^{(7)}$ el año 2003 reportaron un LG infiltrante en la región geniana. Kim et al..$^{(8)}$ el año 2009 y Wu et al. ${ }^{\left({ }^{(9)}\right.}$ el año 2006 reportaron LG en el lóbulo profundo de la glándula parótida. Ralli et al. ${ }^{(10)}$ el año 2017 y Singh el al.(11) el año 2014 reportaron LG en la región cervical. Medina et al..$^{(5)}$ el año 2007 reportaron un LG en la región submental. Hakeem et $a^{\left({ }^{(12)}\right.}$ el año 2018 reportaron un LG en el espacio parafaríngeo que se extendía incluso a la región pterigoidea (base anterior de cráneo). Es decir, según los reportes mencionados anteriormente, los LG de cabeza y cuello pueden ubicarse anatomicamente en diferentes regiones e incluso pueden comprometer la estética facial, estructuras glandulares, la vía área, entre otras. En nuestro caso, por su ubicación anatómica la lesión provocaba alteraciones desde el punto de vista estético generando una considerable asimetría hemifacial.

La mayoría de las lesiones lipomatosas no representan ningún desafío desde el punto de vista diagnóstico; sin embargo, es importante hacer la diferenciación con los liposarcomas debido a su agresividad y abordaje terapéutico(13). Los liposarcomas tienen casi la misma presentación clínica que los lipomas benignos, ya que son de consistencia comparable y tampoco producen dolor a la palpación. A veces, incluso su presentación radiográfica también es similar. Es por esto la importancia de hacer siempre el diagnóstico diferencial, considerando la velocidad de crecimiento de la masa tumoral, su aspecto imageneológico e histopatología. Una lesión adherida a planos profundos, de crecimiento rápido y que mide más de 10 centímetros de diámetro es sugerente de lesión maligna ${ }^{(14)}$. En nuestro caso, la tumoración no se encontraba adherida a los planos profundos (móvil). Su evolución era de larga data ( 9 años) y no se asociaba a adenopatías regionales palpables sugerentes de liposarcoma.

Si bien la ecografía y la TC con medio de contraste a menudo pueden sugerir un diagnóstico de lipoma, la resonancia nuclear magnética es el estudio imageneológico de elección, ya que puede confirmar la presencia de un lipoma o revelar características atípicas indicativas de liposarcoma u otro tipo de lesión. Una masa muy grasa con señales hiper-intensas de T1 y T2, pocos o ningún septo delgado y áreas mínimas o nulas de crecimiento sugiere un diagnóstico pre-operatorio de lipoma. Algunos lipomas pueden mostrar septos fibrosos prominentes y nodularidad en la resonancia magnética, imitando la aparición de liposarcomas bien diferenciados. Una secuencia de resonancia magnética de saturación de grasa es altamente efectiva para evaluar las lesiones intra-tumorales en tales casos. Una vez que los datos de imágenes se han interpretado correctamente, la masa se puede extirpar quirúrgicamente. Un examen histopatológico post-quirúrgico proporciona el diagnóstico definitivo10. En nuestro caso, el examen clínico, la TC pre-quirúrgica y la PAAF nos sugirieron fuertemente un diagnóstico inicial de lipoma. El cual se 
confirmó finalmente con el estudio histopatológico post-quirúrgico.

El tratamiento de elección de los LG es la exéresis quirúrgica de la tumoración junto con su cápsula. Es necesario un minucioso conocimiento de la anatomía y de las diferentes técnicas reconstructivas a la hora de abordar quirugicamente un LG, para así conseguir resultados funcionales y estéticos satisfactorios. Aunque tradicionalmente se han empleado incisiones a lo largo de las líneas de tensión de la cara, debemos emplear abordajes que permitan ocultar y minimizar las cicatrices quirúrgicas ${ }^{(15)}$ En nuestro caso, se realizó la exéresis completa de la lesión respetando su cápsula y las estructuras anatómicas vecinas mediante abordajes intra y extraorales.

\section{CONCLUSIONES}

Para el diagnóstico adecuado de LG es importante tener en consideración todos los aspectos clínicos, imagenológicas e histopatológicos de esta lesión.

Debido a que los LG de cabeza y cuello son infrecuentes, pueden confundirse con otras lesiones, por lo que es importante hacer el diagnóstico diferencial.

Los LG de cabeza y cuello pueden tener diferentes ubicaciones anatómicas e incluso comprometer la funcionalidad y éstetica es estadíos avanzados. Una minuciosa planificación pre-quirúrgica y un conocimiento acabado de la anatomía son fundamentales para su correcto manejo terapéutico.

\section{CONFLICTO DE INTERESES}

Los autores declaran que no tienen ningún conflicto de intereses.

\section{FUENTES DE FINANCIAMIENTO}

Este trabajo no cuenta con financiamiento alguno.

\section{Bibliografía}

1. Som PM, Scherl MP, Rao VM, Biller HF. Rare presentations of ordinary lipomas of the head and neck: a review. AJNR Am J Neuroradiol. 1986;7(4):657-64.

2. El-Monem MH, Gaafar AH, Magdy EA. Lipomas of the head and neck: presentation variability and diagnostic work-up. J Laryngol Otol. 2006;120(1):47-55.

3. Kim JS, Choi MS, Lee SJ, Park BC. Clinico-surgical characteristics of head and neck lipomas. Dermatol Surg. 2018;44(6):893-5.

4. Copcu E, Sivrioglu N. Posterior cervical giant lipomas. Plast Reconstr Surg. 2005;115(7):2156-7.

5. Medina CR, Schneider S, Mitra A, Spears J, Mitra A. Giant submental lipoma: Case report and review of the literature. Can J Plast Surg. 2007;15(4):219-22.

6. Signorini M, Campiglio GL. Posttraumatic lipomas: where do they really come from?. Plast Reconstr Surg. 1998;101(3):699-705.

7. Salvatore C, Antonio B, Del Vecchio W, Lanza A, Tartaro G, Giuseppe C. Giant infiltrating lipoma of the face: CT and MR imaging findings. AJNR Am J Neuroradiol. 2003;24(2):283-6.

8. Kim DS, Kwon H, You G, Jung SN. Surgical treatment of a giant lipoma in the parotid gland. J Craniofac Surg. 2009;20(5):1601-2.
9. Wu CW, Chi HP, Chiang FY, Hsu YC, Chan LP, Kuo WR. Giant lipoma arising from deep lobe of the parotid gland. World J Surg Oncol. 2006;4:28.

10. Ralli M, de Vincentiis M, Greco A. First, rule out cancer: giant lipoma. Am J Med. 2018;131(2):146-7

11. Singh M, Saxena A, Kumar L, Karande SK, Kolhe Y. Giant lipoma of posterio cervical region. Case Rep Surg. 2014;2014:289383.

12. Hakeem AH, Hakeem IH, Budharapu A, Wani FJ. Giant parapharyngeal space lipoma extending to the pterygoid region (anterior skull base). J Craniofac Surg. 2018;29(2):e149-e150.

13. Minić AJ. Well-differentiated liposarcoma mimicking a pleomorphic lipoma--a case report. J Craniomaxillofac Surg. 1993;21(3):124-6.

14. Jones AP, Lewis CJ, Dildey P, Hide G, Ragbir M. Lipoma or liposarcoma? A cautionary case report. J Plast Reconstr Aesthet Surg. 2012;65(1):e11-e14.

15. Cillo JE Jr, Caloss R, Wendelken JA. Excision of subcutaneous facial cysts and lipomas using cosmetic approaches. J Oral Maxillofac Surg. 2006;64(11):1603-16. 